\title{
DE L'EXPÉRIENCE DE VIE À LA MISE EN DISCOURS DU VÉCU: QUE PEUT NOUS APPRENDRE UNE HERMÉNEUTIQUE DE LA PAROLE?
}

RÉSUMÉ

Le texte discute le phénomène de la parole comme un procédé heuristique permettant d'étudier comment les dynamiques vitales et les dynamiques du vivant se révèlent chez un sujet comme manière de signifier son être-là, dans la présence d'un ici et maintenant. La spécificité de ce travail consiste à mettre en dialogue l'anthropologie avec les apports de la philosophie herméneutique: le problème de la construction des savoirs basés sur le recueil du discours des acteurs apparaît alors sous les auspices d'un problème général d'interprétation. Le travail herméneutique portant sur l'étude des multiples visées référentielles de la parole consiste à explorer le réseau de relations à partir duquel se compose pour un sujet-parlant, dans le présent vivant, une idée de soi et du monde: la charge sémantique de cette double tension directionnelle jette en même temps un pont vers la phénoménologie et la pragmatique du langage ordinaire. On peut ainsi se demander de quoi la parole est la manifestation? À la faveur de quoi se construit le sens du discours? Comment est parlé ce qui est dit?

Mots-clés: Recherche biographique. Anthropologie. Herméneutique de la parole.

\section{ABSTRACT FROM THE EXPERIENCE OF LIFE TO THE SPEECH ON LIVED EXPERIENCE: WHAT CAN WE LEARN FROM HERMENUTICS OF SPEECH?}

The text discusses the phenomenon of speech as a heuristic process to study how vital dynamics and dynamics of the living are revealed in a subject as a way to signify his being there, in the presence of a here and now. The specificity of this work consists in bringing anthropology into dialogue with the contributions of hermeneutical philosophy: the problem of the construction of knowledge based on the collection of migrant's discourses then appears under the auspices 
of a general problem of interpretation. The hermeneutical work dealing with the study of the multiple referential aims of speech consists in exploring the network of relations composing for a subject-speaking, present, an idea of the self and the world in the present: the semantic charge of this dual directional tension casts a bridge to the phenomenology and pragmatics of language. One can thus ask: what word is manifested? In which direction is the meaning of speech built? How is said what is said?

Kewords: Biographical Research. Anthropology. Hermeneutics of Speech.

\section{RESUMO DA EXPERIÊNCIA DE VIDA AO DISCURSO DO VIVIDO: O QUE PODEMOS APRENDER COM UMA HERMENÊUTICA} DA PALAVRA?

O presente texto discute a palavra enquanto fenômeno heurístico que permite estudar as formas pelas quais se revelam as dinâmicas vitais e as dinâmicas vividas pelos indivíduos enquanto formas de significar o ser em sua presença no aqui e agora. A especificidade deste trabalho consiste em estabelecer um diálogo entre a antropologia, e suas contribuições oriundas da filosofia hermenêutica, para refletir sobre o problema da construção dos saberes baseada na coleta de discursos de indivíduos migrantes e sua consequente interpretação. 0 trabalho hermenêutico relativo aos estudos que assumem a palavra como referência consiste em explorar a rede de relações através das quais o indivíduo, enquanto sujeito de fala no momento presente, se constrói em relação a si mesmo e ao mundo: a carga semântica desta dupla tensão de direção lança, ao mesmo tempo, uma ponte em direção à fenomenologia e à pragmática da linguagem. Assim, algumas questões atravessam esse texto: Qual é a palavra que se manifesta? A favor de que se constrói o sentido do discurso? Como é falado aquilo que é dito?

Palavras-chave: Pesquisa biográfica. Antropologia. Hermenêutica da palavra.

\section{Introduction}

Dans le cadre de ma recherche de thèse doctorale intitulée "Le présent vécu comme processus de formation du sujet anthropologique: une herméneutique de la parole en condition de migration précaire » (GADRAS, 2017), j'ai ob- servé durant quatre années un groupe de migrants en situation dite irrégulière: le collectif des Sorins. Originaires de l'Afrique subsaharienne, ces 173 hommes occupent un squat d'habitation (BOUILLON, 2011) dans la ville de 
Montreuil, en région parisienne (France). L'ancienne usine désaffectée où ils ont trouvé refuge témoigne des politiques de non-accueil auxquelles se confrontent les migrants « indésirables » (AGIER, 2011) à l'échelle de l'Europe, faisant aussi l'épreuve des régimes de précarisation qui en découlent. Pour survivre à ces conditions de grande vulnérabilité sociale, l'organisation du quotidien chez les 'Sorins' repose sur un fonctionnement associatif, ce qui permet de prendre en charge collectivement toutes les préoccupations ayant traits à la survie et à la dignité de chacun de ses membres (GADRAS, 2015). Depuis ce complexe terrain d'investigation où les stratégies de lutte collective se mêlent à des tactiques individuelles et confidentielles, il me paraît impossible de tenir un discours général sur la manière dont chacun de ces migrants interprète le sens de sa propre présence en France au regard de son parcours, de ses situations de vie et de ses motivations. Pour étudier la manière dont les participants de la recherche conçoivent leurs situations de vie et confèrent du sens à leurs actions au moment où ils parlent à quelqu'un, je me suis interrogé au sujet du « pouvoir-imageant » (RICOEUR, 1975) de la parole. Partant de l'idée que l'expérience est, en tant que telle, le produit d'une interprétation (GADAMER, 1996), il m'est apparu primordial de chercher à éclairer ce processus de construction de sens en vue de pouvoir étudier ce qui est porté au langage quand une personne cherche à exprimer la manière dont elle vit sa vie. Pour pénétrer dans cette dimension singulière de l'expérience, il convient d'explorer comment, l'êtreen-soi placé devant les occurrences de son être-au-monde donne forme à la réalité humaine, c'est-à-dire à un pour-soi depuis les possibilités du monde.

À un niveau méthodologique, cette entreprise de recherche nécessita le recours à des conversations de terrain (BECKER, 2002) ainsi qu'à des entretiens de recherche biographique (DELORY-MOMBERGER, 2009). Sur un plan épistémologique, l'enjeu consistait à déterminer s'il était possible de concevoir une investigation de parole capable de rendre compte des processus de mises-en-formes du vécu d'expérience d'un sujet. Mais avant de répondre à ce questionnement, il convient d'expliquer le cheminement de la réflexion qui a donné lieu à l'élaboration d'une proposition méthodologique que j'ai désignée " herméneutique de la parole »(GADRAS, 2017). Expliquer ce que peut nous apprendre une herméneutique de la parole implique, à mon sens, de mettre en exergue les pistes de réflexion qui ont contribué à modeler ce mode heuristique d'investigation.

\section{Le discours, ou le miroir d'une dynamique vivante}

Dans les approches qualitatives en sciences sociales, s'interroger sur la manière dont la parole des acteurs permet ou non d'éclairer un phénomène social est une démarche courante. Les méthodes "d'entretiens ethnographiques » (BEAUD \& WEBER, 1998), tout comme les entretiens biographiques (DEMAZIERE \& DUBAR, 1997) réalisés suivant le principe du « récit de vie » (BERTHAUX, 1976), constituent des modes légitimes d'investigation pour les sociologues et les anthropologues. En effet, un très large consensus scientifique s'accorde autour de l'idée suivant laquelle, le discours des acteurs porte les traces et les signes des phénomènes dans lesquels ils se trouvent impliqués, par le biais de leurs activités sociales. Parallèlement, l'analyse de ce matériau empirique (le discours) aura contribué aussi à mettre en évidence qu'en entretien les acteurs tendent à réinterpréter les situations sociales qu'ils décrivent, ce qui suppose l'existence d'un effet de distorsion entre le discours et la 
réalité. Procédant de ce constat, de sévères critiques et procès de mise-en-garde ont rejailli sur la fiabilité du discours dans la construction des savoirs en sciences sociales.

Avec l'opposition dichotomique et théorique de la subjectivité et de la scientificité émerge cette problématique générale avec des variantes liées aux disciplines de recherche: comment intégrer à une démarche scientifique un matériau foncièrement " subjectif »? En sociologie qualitative et en anthropologie, les soupçons à l'égard du discours induisent la mise en œuvre de stratégies d'ordre méthodologique: sujet à caution, le contenu textuel des énoncés est subordonné à une logique de superposition et de recoupement où le chercheur met en balance les traces d'activités qu'il a recueillies - au moyen de l'observation, de la description et de l'archivage notamment - avec ce qui est dit par les acteurs; ce travail de mise à distance permet d'objectiver les infléchissements subjectifs du discours où s'évanouit de ce fait une part de sa crédibilité. Sans venir ignorer ou nier, pour autant, ce que les sujets cherchent à exprimer, ce complexe travail d'analyse et d'objectivation confère une rationalité scientifique ${ }^{1}$ (ici comprise au sens positiviste du terme) à cette approche qualitative de la recherche. Cela semble aller de soi ! Pourtant, le principe de ce raisonnement n'atténue en rien le clair-obscur qui entoure le discours dans son rapport à la réalité: en effet, force est de constater que cette conception du discours apparaît dans les faits sous les occurrences d'un " obstacle épistémologique » (BACHELARD, 1938), au sens où le point de vue subjectif des acteurs engendre des distorsions de la réalité (le « Réel », diront certains) qui entravent l'accès à une compréhension immédiate du phénomène. Tout se passe en fait

1 Le discours de acteurs, leur parole, est en ce cas renvoyé au problème général de la construction de la science: produire de la connaissance reposant sur des énoncés qui soient justes et vérifiables. comme si le contenu d'un discours n'a de sens que lorsqu'il tend à rendre compte d'un cadre tangible d'existence car, dans le fond, que faire des 'bons sentiments' des sujets, sans verser dans la psychanalyse ou la psychologie. Quand, du reste, la réalité est conçue comme un phénomène existant en dehors de l'homme, que faire des inférences subjectives et singulières qui incarnent la matérialité et la concrétude d'une puissance de vie, dont témoigne le rapport de coappartenance qui unit le sujet-parlant à l'expérience qu'il fait du monde?

Ce processus vital - l'être au monde - appelle à se questionner sur les conditions mêmes de réalisation des vies humaines ainsi que sur leurs effets dans la vie quotidienne d'un sujet. Quelle est la fonction du connaître produit par la science dans son rapport à l'expérience de la vie et du monde faite par des sujets-humains? Comment l'homme depuis ses conditions d'exister construit-il son rapport au monde? Qu'est-ce que vivre fait à l'homme?

\section{La fonction heuristique de la parole}

C'est à la faveur de cette réflexion critique tournée vers le délicat statut du discours dans les approches dites qualitatives que débute mon investigation sur la notion de parole - et l'on voudra bien m'excuser d'en avoir grossi le trait. En ce sens, mon travail de thèse en recherche biographique, interroge comment « l'expression vive dit l'existence vive » (RICOEUR, 1975, p. 61), en examinant notamment « le mouvement réflexif et vital qui relie l'être-au-monde [et] se déploie à la faveur d'activité d'interprétation (DELORY-MOMBERGER, 2009) » (GADRAS, 2017, p. 53) et la manière dont le discours s'affirme chez un sujet comme manière de voir le monde et de le comprendre. L'idée selon laquelle il y a « quelque chose d'énergique et de vital dans le langage qui ne se laisse pas 
réduire à des 'messages' » (LAPLANTINE, 2005, p. 200) annonce la ligne directrice d'une entreprise épistémologique et méthodologique dont l'efficace s'incarne, à mon sens, dans la vigueur de ce questionnement « que nous montre la parole? » (HEIDEGGER, 1957). Sans effet de rhétorique, la formule invite à porter une attention accrue sur la parole en interrogeant sa fonction au sein même de son propre déploiement. Ainsi, la façon dont parle la parole ne renvoie pas tant à un texte qu'à un « chemin », nous dit Heidegger (1959). Et la portée de son heuristicité s'ouvre à la possibilité de connaître et de comprendre la manière dont un sujet-humain s'ouvre à sa propre existence (GADRAS, 2017). Suivant cette approche, le pouvoir heuristique de la parole " comporte une double tension directionnelle, l'être et le monde » (Ibid, p. 129), et le fond commun de ce rapport tensionnel est l'expérience éprouvante qu'un sujet fait de sa vie, que ce soit au plan individuel, collectif ou social. Autrement dit, le potentiel heuristique de la parole s'offre dans le réfléchissement du mouvement par lequel un sujet s'ouvre à la possibilité de se comprendre et de se (re)connaître dans le monde. (En tant qu'il se manifeste comme un acte d'auto-interprétation engendrant une donation de sens, ce phénomène anthropologique est désigné par Delory-Momberger (2003; 2009) travail biographique. Par conséquent, la parole est révélatrice d'une activité d'écriture et de composition en ce qu'elle porte en ellemême un pouvoir de description des modes de production de sa charge sémantique (la compréhension de soi) ainsi que la visée réelle de sa détermination (la référence au monde), se caractérisant par la donation de sens d'un sujet qui ainsi confère au texte sa portée signifiante et singulière.

Dans la perspective de cette approche, la « réalité » humaine dans son rapport à la parole et au discours - en deçà de l'opposition que l'on retrouve chez les positivistes entre l'émotion et les faits - porte sur « un état de chose, c'est-à-dire un ensemble de relations qui lient les choses entre-elles à travers [des actions] et des faits " (GADRAS, 2017, p. 239). Par exemple, 'se sentir bien' n'est pas un état d'âme propre à un individu, mais constitue un archétype assignable à l'ensemble du genre humain, en tant qu'il témoigne d'une disposition à être en lien avec une idée ou une chose adéquate. Ainsi, à l'instar du paradigme de l'anthropologie modale, dont l'objectif consiste à saisir les dynamiques du vivant pour étudier « la vie en train de se faire » (LAPLANTINE, 2005, pp. 190-216), le « Réel » ici se conçoit comme le vivant « en perpétuelle vibration » (Ibid, p. 196). L'une des implications immédiates de ce raisonnement consiste à prendre au sérieux cette réalité anthropologique radicale l'être humain entre dans le mouvement de la vie et du monde à partir des réalités sensibles qui le constituent et lui permettent d'aborder le monde et d'agir sur lui (HONORE, 2005), en conférant des significations à son être-là (DELORY-MOMBERGER, 2009). De ce point de vue, une herméneutique de la parole vise à explorer les dynamiques du vivant qui engendrent chez un sujet-humain " une présence à soi dans le présent du présent vivant » (DERRIDA, 1967, p. 8), c'est-à-dire, une conscientisation de son être-là par la formation d'une idée de soi et du monde.

Si la manière d'aborder le monde d'un sujet-humain est solidaire des réalités sensibles desquelles procède le mode du comprendre alors le déploiement de la parole montre potentiellement comment se tissent les relations entre le « vivant », le « vital » et le « vécu ».

\section{Le dire et le parler}

Dans sa réflexion autour de la philosophie herméneutique, Ricoeur propose une définition exhaustive du discours qu'il décline ain- 
si « le discours articule un sujet de discours, un acte de discours, un contenu de discours, un code méta-linguistique, une référence extra-linguistique, un interlocuteur » (RICOEUR, 2010 , p. 36). On remarquera immédiatement que cette proposition pointe dans le discours l'existence d'une dimension méta-linguistique et extra-linguistique, dont témoigne " le corps engagé dans le langage [en raison de l'] activité à la fois physique, émotionnelle et cognitive d'un sujet " (LAPLANTINE, 2005, p. 203). Le discours repose sur différents fondements et jusqu'ici la vigilance du chercheur s'est essentiellement portée sur l'acte de discours, le contenu du discours et les situations d'interlocution. Déjà, au cœur de sa réflexion sur la parole qui déboucha sur une série de conférences, Heidegger avertissait de cette insuffisance en déplorant, d'une certaine façon, que l'attention portée au discours (au dire de la parole) conduise à ignorer l'expérience de l'être-là dont témoigne la parole. Ainsi, dans ses pérégrinations sur la parole, il établit une distinction entre le dire et le parler d'un côté, « dire signifie: montrer, laisser apparaitre, présenter (Darreichen) un monde [...] » (HEIDEGGER, [1957] 2014, p. 200); et de l'autre, " le parler appelle les choses au monde; il appelle en présence le monde » (Heidegger, [1950] 2014, p. 27), au sens où la temporalité du parler coïncide avec une manière d'aborder le monde, de le voir et de le sentir, soit de lui donner une forme.

Avec le dire, la structure de déploiement de la parole s'oriente vers ce qui est dit explicitement (au plan linguistique et lexicographique), quand le parler suggère quant à lui un mode de présence (au niveau existential et énergétique). C'est pourquoi, le parler montre comment l'homme s'ouvre à son existence, et le dire montre comment la parole conquiert sur les bases d'un lexique commun (le langage), sa signification singulière.

\section{La corporéité de la parole}

En quoi la parole d'un sujet vient-elle à rendre compte de l'épreuve éprouvante que l'homme fait de son être-au-monde quand il s'ouvre à son existence? Que peut nous apprendre à ce sujet le déploiement de la parole? Pour comprendre comment le parler de la parole ouvre la voie - montre le " chemin » - vers ce qui est corporellement en mouvement, il est capital de considérer que la vie se manifeste chez l'homme en sa forme la plus radicale comme pouvoir de jouir et pouvoir de pâtir de ses affections (SPINOZA, [1677] 2014). Cela signifie que l'homme s'ouvre à son existence en faisant nécessairement l'épreuve de sa propre mise en présence dans le monde. Cette expérience éprouvante dérive, dans la présence du présent vivant, du rapport homme-chose (un objet, des idées-choses, une personne, etc), qui se caractérise chez un sujet dans le jouir ou le souffrir (Honoré, 2005). Spinoza assigne à cette propriété ontologique la dénomination d'affect, au sens où l'affect est une affection du corps et en même temps l'idée de ce corps, en tant qu'il comprend l'idée du corps affecté et du corps affectant (DELEUZE, 1981). Il ne s'agit pas d'un concept psychologique ${ }^{2}$, mais d'un mode existential soit une manière d'être disposé (re-disposé) à aborder le monde, d'une certaine façon, en faisant l'épreuve de ses sentiments, qui résonnent dans la gamme de la joie ou de la tristesse (HONORE, 2005). L'expérience éprouvante de la présence qui engendre chez l'homme une idée de soi et du monde procède de cette disposition par laquelle un sujet peut entendre quelque chose (la disposibilité) de la tonalité de l'épreuve de l'entredeux homme-chose. Le corps anthropologique est le pivot de cette matrice relationnelle, qui attelle l'homme au mouvement de sa propre existence: ce processus vivant comprend à la

2 On retrouve également cette approche conceptuelle chez Heidegger (1927). 
fois les dynamiques vitales, physiologiques et spirituelles qui animent le corps (le conatus ${ }^{3}$, le désir, la volonté, etc) et auxquelles s'enchevêtrent des activités d'interprétation où se " relationne » (HONORE, 2005) le rapport « individu-situation-environnement » (NUTTIN, 1980). Donc, la corporéité de la parole repose sur l'imbrication d'un double processus: l'élan vital dans lequel s'inscrivent les dynamiques vivantes de l'être-corps; et la réflexisation qui naît chez l'homme à partir de l'épreuve qu'il fait de ses affects (HONORE, 2005).

Partant du postulat que le corps est engagé dans le langage quand un sujet parle, il convient de considérer, me semble-t-il, que les changements d'affect et les variations de modes témoignent de la dimension corporelle de la parole (l'expression vive), de même que le déploiement de la parole - en tant qu'il montre un état de relations entre les choses et les faits - témoigne de la puissance de vie et de la puissance d'action du sujet-parlant, c'est-à-dire de son état de l'âme (son mood / son mode d'être) et de sa capacité à agir avec et sur le monde (l'existence vive). Ce pouvoir de dire le monde - « de dire quelque chose à propos de quelque chose » (RICOEUR, 2010, p. 95) - s'enracine dans les médiations du corps par lesquelles s' instituent les conditions d'une présence à soi qui s'incarne ipso facto dans la

3 En résumé (et je fais ici référence à la pensée philosophique de Spinoza), la notion de conatus consiste en l'idée que tout être vivant persévère en son être selon la durée qui lui est propre, c'est-à-dire à travers une continuité indéfinie d'existence (cf chap II, expl. 5). Ce substantif renvoie à deux idées essentielles: aucune vie n'a en elle les causes de sa propre destruction; le conatus est une puissance toujours en acte, qui est inséparable de sa dynamique d'actualisation (cf chap III, prop. 7 et 8). La puissance est donc attachée au conatus de chaque chose et varie chez l'être humain selon " des seuils d'intensité répartis entre un minimum et un maximum, le premier correspondant un pôle d'extrême passivité, le second à un pôle d'extrême activité » (MACHEREY, 1995, p. 24). La variation de ces pôles dérive « des « affects primaires » (affectus primarii), qui sont au nombre de trois: le « désir » (cupiditas), la joie (laetitia) et la tristesse (tristitia) » (Ibid, p. 24). copule du verbe « être $»^{4}($ RICOEUR, 1975) et la forme modale du « Je $»^{5}$ (RICOEUR, 2004). C'est ainsi qu'un examen approfondi de la parole ouvre un « chemin » de compréhension vers la manière dont un sujet aborde sa mondanité et appréhende ses situations de vie, depuis son 'être-là', dans un ici et maintenant.

\section{Le texte public et le texte privé}

Le rapport dialogique qui s'opère entre le dire et le parler laisse apparaître une part du réseau de relations à travers lequel le processus de compréhension de soi de l'être de langage(s) s'incorpore à une idée du monde. De sorte qu'au moment où un sujet 'dit quelque chose à quelqu'un au sujet de quelque chose' son activité interprétante se tourne vers une dynamique de sens dont l'élan repose sur le désir et un souci de soi6 caractérisé par les conditions sociales d'existence. Ce qui signifie qu'au plan social, le déploiement du parler et du dire - au-delà de "l'histoire du corps » (MACHEREY, 1995) et des médiations qui en découlent - se trouve être également déterminé par l'environnement contextuel dans lequel évolue un sujet, au sens où le pouvoir de dire d'un sujet est conditionné par la place qu'il occupe dans le champ des forces qui font le jeu social et les enjeux de reconnaissance qui en dérivent.

En tant qu'il s'arque boute sur une volonté de dire (DERRIDA, 1972), le pouvoir de dire des personnes en situation d'exil peut donc se restreindre à l'injonction de prouver la

4 En tant qu'il dit ce qui est et ce qui n'est pas son lieu le plus propre (l'être-dit) se trouve dans la copule du verbe être (RICOEUR, 1975).

5 Avec la forme modale du « Je », nous retrouvons les quatre figures de la capacité qui dans la phénoménologie de l'homme capable de Ricoeur (2004) se décline comme pouvoir de dire, pouvoir de faire, pouvoir de raconter et de se raconter - et de se reconnaître in fine comme l'auteur d'une action (l'imputabilité).

6 Dans la philosophie de Ricoeur (1990), le Soi n'existe pas par lui-même ou pour lui-même mais se comprend toujours à partir de l'altérité du monde. 
« véracité » de leur récit au regard du mode d'obtention de l'asile en France, qui se base notamment sur l'examen de preuves matérielles (DELORY-MOMBERGER, 2012): le récit est mis à l'épreuve des représentations des membres de l'instance qui évaluent la crédibilité des justifications attestant du péril du demandeur d'asile dans son pays d'origine. Ainsi, la mise en forme du récit constitue un enjeu vital car de son effet performatif dépend le verdict de la commission Ofpra7 et avec lui la vie et l'avenir d'une personne. À partir de ces considérations, il me paraît inévitable de supposer que les nécessités vitales qui soustendent ce type de démarche infèrent, d'une manière ou d'une autre, sur le déploiement de la parole. Ce qui ne veut pas dire que le jeu de création et de mise en scène qui en découle soit le signe d'une comédie, mais plutôt l'expression de la vulnérabilité sociale qui frappe durement les personnes en situation d'exil, dont l'à venir est estimé à l'aune de mesures techniques donnant corps à des politiques de Non-accueil. Autrement dit, le pouvoir de dire qu'il soit limité ou contraint laisse apparaître de véritables " arts de faire » (de CERTEAUX, 1991), rendant ainsi visible la manière dont un sujet appréhende ses situations d'interaction sociale ainsi que leurs effets performatifs sur le déploiement de la parole.

En partant de la pragmatique du langage ordinaire en linguistique, j'en suis venu à m' intéresser, dans le cadre d'un article relatif à mon travail de thèse (GADRAS, 2015), à la réflexion de Scott (2009) visant à mettre en perspective les rapports de force entre le groupe dominant et les dominés à partir des notions de « texte public » et de « texte privé ». En quelque sorte, pour Scott, les subalternes, c'est-à-dire ceux qui sont « acculé[s] à un ensemble de ma-

7 Il s'agit de la commission de l'Office Français de Protection des Réfugiés et Apatrides (OFPRA) où tous les demandeurs d'asile se trouvant sur territoire français doivent adresser leur demande. nières d'être qui sont sans effet sur les conditions dans lesquelles elles apparaissent » (Le BLANC, 2010, p. 99), s'approprient le langage du groupe dominant en vue de transformer les représentations sociales qui les stigmatisent et de favoriser leur inclusion sociale. Le " texte public » est l'expression d'un rapport de force politique qui impose aux dominés d'adopter le comportement social et le discours promu par le groupe dominant. De ce point de vue, le groupe des Sorins épouse, autant que possible, les conduites sociales approuvées par le groupe dominant, dans l'espoir d'être valorisés eux-mêmes en retour $»^{8}$ (GADRAS, 2017, pp. 75-76). Tout comme le déploiement de la parole révèle le mode de présence d'un sujet, le « texte public » témoigne du niveau d'adhésion d'un sujet aux considérations sociales et politiques à partir desquelles une société humaine tend à se reconnaître. À mon sens, le « texte public » s'apparente pour les plus vulnérables à une sorte de rempart : il est donc scandé aussi souvent que cela peut apparaître nécessaire. À l'inverse, le " texte privé » (ou le " texte caché ») est maintenu dans le secret. Son dévoilement donne à entendre les tactiques et les réinventions qui permettent aux subalternes de vivre au quotidien dans les marges du commun des vies ordinaires, tout en cherchant à atteindre finement les objectifs et les finalités qu'ils poursuivent.

\section{Conclusion}

Partant du principe que le sujet-humain est à la fois un être vivant singulier (être-corps) et un être social (être-personne) ayant recours

8 Je fais ici référence au mode de vie collectif particulièrement structuré qui a été adopté par le groupe des "Sorins » en prenant une forme associative permettant d'intégrer à la gestion de leur refuge des habitants de la ville qui les soutienne dans leurs actions depuis 8 ans. Ce mode de fonctionnement est autant apprécié par les acteurs sociaux que les pouvoirs publics (la mairie et la Préfecture) qui observent et parfois soutiennent des initiatives menées par le groupe. 
à des langages pour faire signifier son expérience du monde, le pouvoir heuristique de la parole suppose l'existence d'un rapport symétrique entre l'expérience de la vie et la mise en discours du vécu. De ce point de vue, le déploiement de la parole donne à voir la manière dont un sujet compose avec le monde à partir de ses propres conditions d'existence. Ce processus de formation anthropologique compris comme l'ouverture de l'homme à une possible compréhension de soi, entre en rapport avec le phénomène de la parole par le biais de ce questionnement général: qu'estce que vivre fait à l'homme? L'étude du sens et de la référence dans la circonscription du texte est un problème proprement herméneutique, où l'individuel (l'être-corps), le social (l'être de langage) et la situation d'un sujet confronté aux conditions de sa mondanité provoquent l'émergence d'une idée de soi et du monde.

Le rapport dichotomique subjectivité/objectivité est renvoyé par ce biais à l'étude des processus de subjectivation, c'est-à-dire aux modes de rationalisation des médiations du corps et de la pensée par le langage. De ce fait, un document, un objet ou une trace n'existent pas en dehors des discours qui en configurent les réalités. Ainsi, le contenu du discours ne constitue pas, en tant que tel un élément objectif, mais un propos rationalisé régi par des règles et des codifications déterminées par sa finalité. Sa valeur performative est, en quelque sorte, proportionnelle à son efficience pratique au sein des univers symboliques et physiques où une société humaine vient à reconnaître la valeur ou le rôle d'un document ou d'un objet (une politique de migration et un titre de séjour, par exemple). De ce point de vue, même un objet matériel mis en présence d'un observateur n'en est plus un, mais devient une chose qui de fait échappe à toute prétention d'objectivité (de méta-objectivité). Car même en l'absence de toute référence (ce à quoi peut servir l'objet) la mise en présence suggère par induction ou déduction la construction d'une signification (AFFERGAN, 2015), au sens où la présence interpelle l'esprit humain, qui ne peut atteindre cet objet qu'à partir de son expérience existentielle : une manière de voir et d'interpréter selon un mode d'être culturellement et socialement déterminé. L'objet est ainsi associé à une idée qui elle-même se rapporte à l'idée de l'idée (le téléphone est un outil de communication; le pot sur le table peut contenir des fleurs à moins que ce ne soit un pichet, etc.).

La valeur d'angle de cette perspective épistémologique renvoie la parole à « un pouvoir de se référer à des réalités en dehors du langage »(RICOEUR, 1975, p. 10). Ainsi, sous les auspices du dire, le déploiement de la parole achemine des contenus codifiés par les règles d'usage d'une langue à un niveau linguistique. Pendant que sous le patronage du parler, le déploiement de la parole annonce la manière dont un sujet s'ouvre à sa propre existence et aborde le monde. Il est ici question d'indicatif d'ordre interprétationnel, car si un mode de présence est caractérisé par le souci (c'està-dire un ensemble de préoccupations) et la gamme de résonance à partir de laquelle un être s'ouvre à sa propre existence (la joie ou la tristesse), la puissance sémantique du dire repose sur la tension de l'écart qui se creuse entre la prédication générale (ce que veut dire un mot en dehors de son contexte d'élocution) et la référence suspendue (ce que veut dire un mot, un propos, renvoyé à son contexte d'élocution). En d'autres termes, une herméneutique de la parole permet d'éclairer la manière dont l'expérience éprouvante de la vie se manifeste chez un sujet en ce qu'elle propulse au-devant de l'homme une idée de soi et une idée du monde qui caractérise la puissance de vie du sujet-parlant. 
Au-delà des soubassements du réseau de relations qui unit l'être au monde, une investigation de la parole permet de pointer les conditions d'« invivabilité »(BUTLER, 2014), dont font l'épreuve les personnes les plus vulnérables. Car une herméneutique de la parole ne peut être sourde à la manière dont est parlé ce qui est dit.

\section{Reference}

AFFERGAN, F. Éléments pour une anthropologie de la présence. Revue européenne des sciences sociales, v. 2, n. 53, p. 17-49, 2015. Disponible en: <http:// ress.revues.org/3222>. Accédé à : 15 nov. 2015.

AGIER, M. Le couloir des exilés. Bellecombe-enBauges: Édition du Croquant, 2011.

BACHELARD, G. La formation de l'esprit scientifique. Paris: Vrin, 1938.

BEAUD, S.; WEBER, F. Guide de l'enquête de terrain. Paris: La Découverte, 1998.

BECKER, H. S. Les ficelles du métier. Comment conduire sa recherche en sciences sociales. Paris: La Découverte, 2002.

BERTAUX, D. L'approche biographique : sa validité méthodologique, ses potentialités. Cahiers internationaux de sociologie, v. 69, n. 119, p. 198-225, 1980. Disponible en: <www.persee.fr/ doc/homso 0018-4306 1981 num 5913694 t1 02470000 1>. Accédé à: 10 dec. 2015.

BOUILLON, F. Le squat: problème social ou lieu d'émancipation? Paris: Éditions Rue d'Ulm, 2011.

BUTLER, J. Qu'est-ce qu'une vie bonne? Traduit par Martin Rueff. Paris: Payot/Manuels Payot, 2014.

DE CERTEAU, M. L'invention du quotidien 1. Arts de faire. Paris: Folio Essais, 1991.

DELEUZE, G. Spinoza philosophie pratique. Paris: Minuit, 1981.

DELORY-MOMBERGER, C. La langue coupée. Quand le récit doit faire preuve. In : A. BROSSAT, A.; DEOTTE, $M$. (Dir.). Corps subalternes: migrations, expériences, récits. Paris: L'Harmattan, 2012. p. 159- 172.

DELORY-MOMBERGER, C. La condition biographique. Essais sur le récit de soi dans la modernité avancée. Paris: Téraèdre, 2009.

DELORY-MOMBERGER, C. Biographie et éducation. Figures de l'individu projet. Paris: Anthropos, 2003.

DEMAZIERE, D.; DUBAR, C. Analyser les entretiens biographiques: l'exemple des récits d'insertion. Paris: Nathan, 1997.

DERRIDA, J. La voix et le phénomène. Paris: Quatridge/PUF, [1967] 1993.

DERRIDA, J. Positions. Paris: Les Éditions de Minuit, 1972.

GADAMER, H.-G. Vérités et méthodes. Paris: Seuil, [1966] 1996.

GADRAS, M. Retour sur un terrain en recherche biographique: tours et détours de la rencontre avec le collectif des «Sorins». Le sujet dans la cité, 6, (2), p. 184-194, 2015. Disponible en : <http://www.cairn. info/revue-le-sujet-dans-la-cite-2015-2-page-184. htm>. Accédé à : 10 dec. 2015.

GADRAS, Mike. Le présent vécu comme processus de formation du sujet anthropologique: une herméneutique de la parole en condition de migration précaire. 2017, 419 f. Thèse (Doctorat en Sciences de l'Éducation) - Faculté Sciencies de l'Éducation, Université Sorbonne Paris Cité, Université Paris 13, Paris, 2017.

HEIDEGGER, M. Acheminement vers la parole. Paris: Gallimard. [1950; 1957; 1959] 2014.

HONORE, B. L'épreuve de la présence, essai sur l'angoisse, l'espoir et la joie. Paris: L'Harmattan. 2005.

LAPLANTINE, F. L'anthropologie au coin de la rue. Le social et le sensible. Introduction à une anthropologie modale. Paris: Téraèdre, 2005.

LE BLANC, G. Dedans, dehors. La condition d'étranger. Paris: Seuil, 2010.

MACHEREY, P. Introduction à l'éthique de Spinoza. Paris: PUF, 1995. 
NUTTIN, J. Théorie de la motivation humaine. Paris: $\quad$ tiques. Paris: Seuil, 2010. PUF, 1980.

RICOEUR, P. La métaphore vive. Paris: Seuil, 1975.

RICOEUR, P. Soi-même comme un autre. Paris: Seuil, 1990.

RICOEUR, P. Parcours de la reconnaissance. Paris: SCOTT, J. C. La domination et les arts de la résistance. Fragments d'un disvours subalterne. Paris: éditions Amsterdam, 2009.

SPINOZA. L’Éthique. Paris: Gallimard, [1677] 1954.

Folio Essais, 2004.

Recebido em : 10.01 .2018

RICOEUR, P. Écrits et conférences 2. Herméneu-

Aprovado em : 22.03.2018

Mike Gadras est Docteur en sciences de l'éducation de l'Universite de Paris 13 - Sorbonne Paris Cité. Habilité aux fonctions de maître de conférence en anthropologie. Chercheur associé au laboratoire EXPERICE (Université Paris 13 - Paris 8). Ingénieur de recherche à l'université de Cergy-Pontoise. Laboratoire BONHEURS - ESPE / Site Bernard Hirsh /TechEduLab.e-mail: mike.gadras@gmail.com

Avenue Bernard Hirsch, 95000 Cergy.

+33664135371 